\title{
Two-dimensional and three-dimensional T2 weighted imaging-based radiomic signatures for the preoperative discrimination of ovarian borderline tumors and malignant tumors
}

\author{
Xuefen Liu' ${ }^{1}$, Tianping Wang ${ }^{1}$, Guofu Zhang ${ }^{1}$, Keqin Hua ${ }^{2}$, Hua Jiang ${ }^{2}$, Shaofeng Duan ${ }^{3}$, Jun Jin ${ }^{4}$ and He Zhang ${ }^{1 *}$
}

\begin{abstract}
Background: Ovarian cancer is the most women malignancy in the whole world. It is difficult to differentiate ovarian cancers from ovarian borderline tumors because of some similar imaging findings.Radiomics study may help clinicians to make a proper diagnosis before invasive surgery.

Purpose: To evaluate the ability of T2-weighted imaging (T2WI)-based radiomics to discriminate ovarian borderline tumors (BOTs) from malignancies based on two-dimensional (2D) and three-dimensional (3D) lesion segmentation methods.

Methods: A total of 95 patients with pathologically proven ovarian BOTs and 101 patients with malignancies were retrospectively included in this study. We evaluated the diagnostic performance of the signatures derived from T2WIbased radiomics in their ability to differentiate between BOTs and malignancies and compared the performance differences in the 2D and 3D segmentation models. The least absolute shrinkage and selection operator method (Lasso) was used for radiomics feature selection and machine learning processing.

Results: The radiomics score between BOTs and malignancies in four types of selected T2WI-based radiomics models differed significantly at the statistical level $(p<0.0001)$. For the classification between BOTs and malignant masses, the 2D and 3D coronal T2WI-based radiomics models yielded accuracy values of 0.79 and 0.83 in the testing group, respectively; the 2D and 3D sagittal fat-suppressed (fs) T2WI-based radiomics models yielded an accuracy of 0.78 and 0.99 , respectively.

Conclusions: Our results suggest that T2WI-based radiomic features were highly correlated with ovarian tumor subtype classification. 3D-sagittal MRI radiomics features may help clinicians differentiate ovarian BOTs from malignancies with high ACC.
\end{abstract}

Keywords: Ovarian neoplasm, Magnetic resonance imaging, Computer-Assisted Diagnosis, Radiomics

*Correspondence: zhanghe1790@fckyy.org.cn

1 Department of Radiology, Obstetrics and Gynecology Hospital, Fudan University, Shanghai, P.R. China

Full list of author information is available at the end of the article

\section{Background}

Ovarian borderline tumors (BOTs) account for approximately $10-15 \%$ of epithelial ovarian tumors, with an annual prevalence of $1.8-4.8 / 100,000$ women worldwide [1]. Compared with other ovarian malignant tumors, ovarian BOTs often occur in young patients with earlystage disease, and patients have a good prognosis with original author(s) and the source, provide a link to the Creative Commons licence, and indicate if changes were made. The images or other third party material in this article are included in the article's Creative Commons licence, unless indicated otherwise in a credit line to the material. If material is not included in the article's Creative Commons licence and your intended use is not permitted by statutory regulation or exceeds the permitted use, you will need to obtain permission directly from the copyright holder. To view a copy of this licence, visit http://creativecommons.org/licenses/by/4.0/. The Creative Commons Public Domain Dedication waiver (http://creativeco mmons.org/publicdomain/zero/1.0/) applies to the data made available in this article, unless otherwise stated in a credit line to the data. 
fertility-sparing conservative treatments $[2,3]$. Therefore, preoperative identification of patients with ovarian lesions suspected of being BOTs may be helpful in their management.

Magnetic resonance imaging (MRI) has many advantages in determining the etiology of ovarian masses and is widely used in clinical centers [4] . MRI has high diagnostic performance in differentiating between ovarian benign tumors and malignant tumors [5-9] . Considering the ability to discriminate BOTs from malignant epithelial ovarian tumors, conventional MRI varies with a sensitivity of $58 \%$ to $100 \%$ and a specificity of $61 \%$ to $100 \%$, respectively $[7,10-13]$. Functional MRI (for example, dynamic contrast-enhanced MRI, diffusion-weighted imaging and MR spectroscopy) showed a higher ability to distinguish a BOT from ovarian epithelial cancer than conventional MRI, such as T1-weighted imaging (T1WI) and T2-weighted imaging (T2WI), as shown in recently published studies [11, 12]. However, given that functional MRI acquisition is not routinely used in clinical scenarios, the scanning parameters are not presently standardized universally and may change across MRI machines or institutions. Gross morphological characteristic imaging features appreciated on T1WI and T2WI still have better applicability in the differentiation of BOTs from other malignancies.

As a research hotspot, radiomics is defined as a new 'data-driven' approach for extracting large sets of quantitative signatures from radiological images and shows its potential application in medicine [14, 15]. MR-based radiomic signatures has been shown to help to categorize tumor subtypes and assess tumor presence, spread, recurrence or response to treatment in female cancer patients [16-21]. To date, there have been limited MRI radiomics studies concerning ovarian $\mathrm{BOT}$ and epithelial cancer categorization. The purpose of this research was two-fold: first, we planned to evaluate the diagnostic performance of the MRI radiomics model in discriminating ovarian BOTs from malignancies; second, we sought to clarify whether three-dimensional MR-based radiomic signatures (of the whole lesion) could show better discriminative performance than two-dimensional radiomic signatures (of the maximum lesion) could in the same study sample.

\section{Patients and methods}

\section{Patients}

Our institutional review board (Gynecological and Obstetric Hospital, School of Medicine, Fudan University, Shanghai, China) approved this retrospective study, and the requirement for informed consent was waived for all participants. From January 2014 to December 2017, 438 consecutive patients with clinically suspected gynecological diseases were retrospectively retrieved from our institutional picture archiving and communication system (PACS, GE). The inclusion criteria were as follows: 1) patients with no previous pelvic surgery; 2) patients with no previous gynecological disease history; and 3) patients who had MRI examinations performed at our institution before pelvic or laparoscopic surgery. The exclusion criteria were as follows: 1) patients with previous pelvic surgical history or radiation history; 2) patients whose MRI data were unavailable either due to the examination being performed at another institution or due to claustrophobia; or 3) patients whose data lacked histological results. A total of 91 patients (average age, $39.8 \pm 14.9$ years) with pathologically proven ovarian borderline tumors and 105 patients with ovarian malignancies (average age, $51.9 \pm 12.1$ years) were selected as the study sample for signature selection (Table 1). The information on FIGO stage, pathological type, immunohistological staining results, and laboratory tests were collected through a hospital information system.

\section{MR image acquisition and lesion segmentation and radiomics feature selection}

MRI was performed using a 1.5-T MR system (Magnetom Avanto, Siemens) with a phased-array coil. The routine MRI protocols used to assess pelvic masses included axial turbo spin-echo (TSE) T1-weighted imaging (T1WI), coronal TSE T2-weighted imaging (T2WI), and axial/sagittal TSE fat-suppressed T2WI (fs-T2WI). All lesion segmentation was performed by an experienced radiologist (H.Z.). The lesion segmentation on MRI was manually outlined using ITK-SNAP software (ITK-SNAP, version 3.4.0, www.itksnap.org) (Fig. 1). Two segmentation methods were used in this study: maximum lesion segmentation (two-dimensional, 2D) and whole-lesion segmentation (three-dimensional, 3D) on both sagittal fs-T2W images and coronal T2W images.

Table 1 The summary of the pathological types and numbers of the selected samples

\begin{tabular}{|c|c|c|}
\hline Pathological type & Numbers & Age (yrs.) $)^{a}$ \\
\hline Ovarian borderline tumor & 91 & $39.75 \pm 14.85$ \\
\hline Ovary malignancies & 105 & $51.91 \pm 12.05$ \\
\hline Endometroid cancer & 3 & $44.67 \pm 6.02$ \\
\hline Low-grade adenocarcinoma & 3 & $42.33 \pm 19.96$ \\
\hline Clear cell type & 5 & $49.4 \pm 10.33$ \\
\hline High-grade serous carcinoma & 83 & $52.93 \pm 11.28$ \\
\hline Mucinous carcinoma & 7 & $50 \pm 16.33$ \\
\hline Mixed carcinoma & 4 & $50 \pm 7.65$ \\
\hline Total & 196 & $46.26 \pm 14.71$ \\
\hline
\end{tabular}

${ }^{a}$ mean \pm standard deviation 

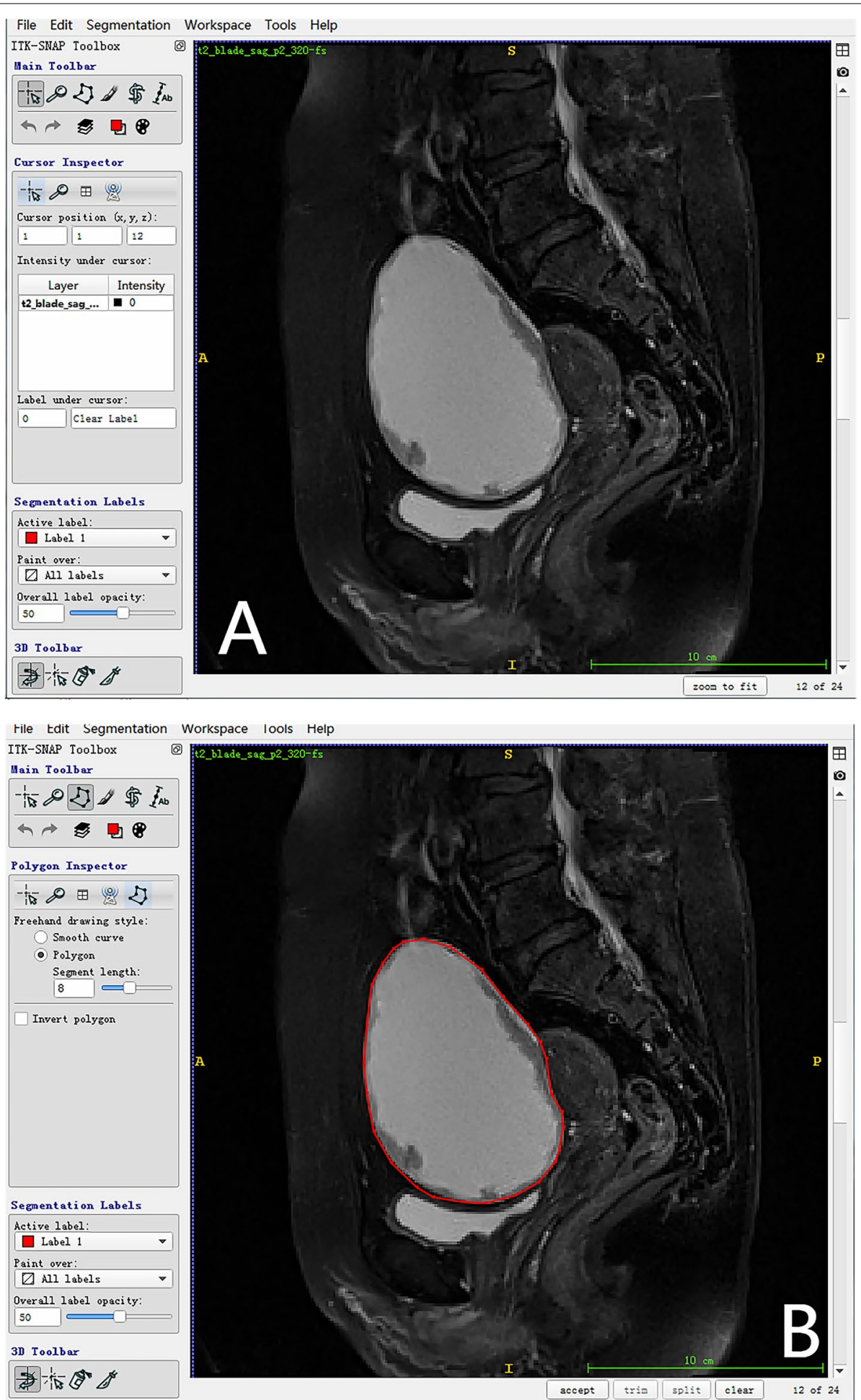

Fig. 1 A 58-year-old woman with pathologically proved high-grade serous carcinoma. We selected the maximum lesion slice on sagittal fs-T2WI and segmented manually along the lesion margin with segmentation tool on ITK-SNAP software. The original fs-T2WI image (A) and region of interest selected image (B) 
In $2 \mathrm{D}$ segmentation, we chose one slice with the largest lesion diameter in two protocols as the premium picture for segmenting the whole lesion. In 3D segmentation, the entire lesion from both protocols was outlined and segmented slice by slice. After the tumor segmentation process, MR-based radiomics signatures were extracted from 2D/3D sagittal fs-T2W and 2D/3D coronal T2W images using AK software on a personal computer (Fig. 1).

\section{Image feature extraction and selection}

A total of 396 radiomics features from the volume of interest were extracted automatically using in-house software (Analysis Kit, version 3.0.0, GE Healthcare). Thereafter, the whole dataset was randomly divided into two parts: a training cohort and a testing cohort. The radiomics score-based signatures were constructed with the least absolute shrinkage and selection operator (LASSO) method, which was used to select the most useful prognostic features in the training data set. A radiomics score (Rad-score) was computed for each patient through a linear combination of selected features weighted by their respective coefficients. These radiomics scores were first assessed in the training data set and then validated in the testing data set.

\section{Statistical analysis}

First, two-sample t-tests were performed to compare MR-based signature values between ovarian BOT and ovarian cancer. Next, the sensitivity (SEN), specificity (SPE), positive predictive value (PPV), and negative predictive value (NPV) were calculated when the performance of the two methods was evaluated for their ability to identify ovarian malignancies. Additionally, receiver operating characteristic (ROC) curve analysis was performed to evaluate various MR-based signature diagnostic values in discriminating BOTs from malignancies. A value of $p<0.05$ was considered statistically significant.

\section{Results}

\section{Clinical characteristics in both the training and testing data sets}

In this study, we included 91 ovarian borderline tumors and 105 ovarian malignancies (83 serous epithelial carcinomas, 7 mucinous carcinomas, 4 mixed carcinomas, 5 clear cell type carcinomas, 3 endometrioid carcinomas and 3 low-grade carcinomas, Table 1). There was no statistically significant difference found between the training and the validation data set in either clinical characteristics or pathological subtypes (Table 2).

Table 2 Clinical and pathological data summaries in both training and testing cohort

\begin{tabular}{|c|c|c|c|}
\hline & Training group $(N=99)$ & Testing group $(N=97)$ & $P$ value \\
\hline Age (yrs.) & $45.9 \pm 13.35$ & $46.64 \pm 15.90$ & 0.961 \\
\hline$<30$ & $17(17.2 \%)$ & $14(14.4 \%)$ & \\
\hline $30-50$ & $35(35.4 \%)$ & $40(41.2 \%)$ & \\
\hline$>50$ & $47(47.5 \%)$ & $43(44.3 \%)$ & \\
\hline Ki-67 expression (\%) & $32.37 \pm 28.01$ & $25.05 \pm 26.35$ & 0.946 \\
\hline$<50$ & $59(67.0 \%)$ & $74(84.1 \%)$ & \\
\hline $50-75$ & $20(22.7 \%)$ & $5(5.7 \%)$ & \\
\hline$>75$ & $9(10.2 \%)$ & $9(10.2 \%)$ & \\
\hline CA-125 level(IU/L) & $553.32 \pm 994.28$ & $300.30 \pm 452.27$ & 0.000 \\
\hline$<35$ & $15(22.7 \%)$ & $18(27.7 \%)$ & \\
\hline $35-200$ & $17(25.8 \%)$ & $24(36.9 \%)$ & \\
\hline $200-500$ & 13(19.7\%) & $10(15.4 \%)$ & \\
\hline$>500$ & $21(31.8 \%)$ & $13(20.0 \%)$ & \\
\hline Category & & & 0.980 \\
\hline Borderline tumor & $47(47.5 \%)$ & $44(45.4 \%)$ & \\
\hline Malignancies & $52(52.5 \%)$ & $53(54.6 \%)$ & \\
\hline Endometroid cancer & $2(2.0 \%)$ & $1(1.0 \%)$ & \\
\hline Low-grade adenocarcinoma & $0(0.0 \%)$ & $3(3.1 \%)$ & \\
\hline Clear cell type & $1(1 . \%)$ & $4(4.1 \%)$ & \\
\hline Serous carcinoma & $45(45.5 \%)$ & $38(39.2 \%)$ & \\
\hline Mucinous carcinoma & $2(2.0 \%)$ & $5(5.2 \%)$ & \\
\hline Mixed carcinoma & $2(2.0 \%)$ & $2(2.1 \%)$ & \\
\hline
\end{tabular}




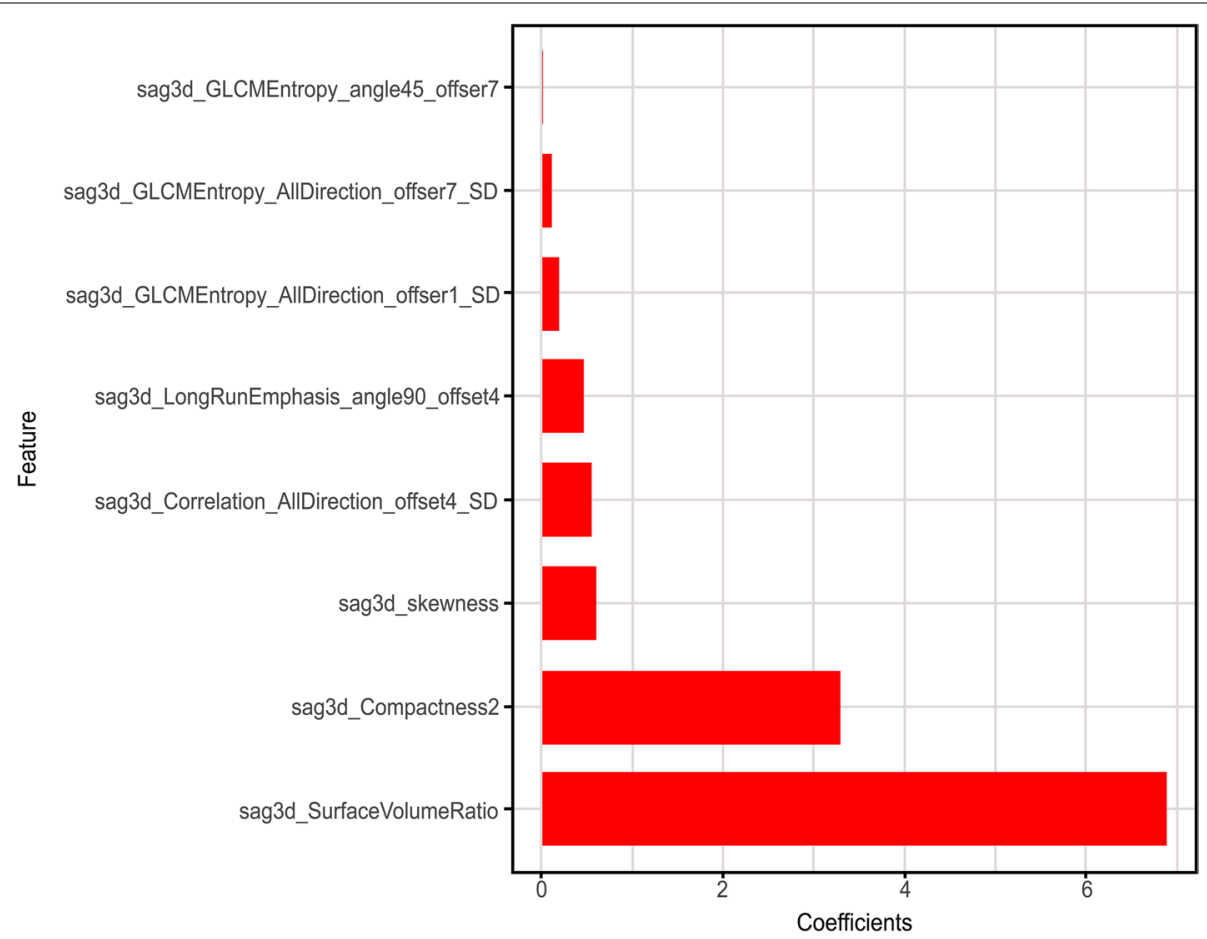

Fig. 2 Histogram shows the weight of various features that contribute to the 3D signatures on sagittal fs-T2WI. The features that contribute to the radiomics signature model are displayed on the $y$-axis, with their coefficients in the LASSO analysis model dotted on the $x$-axis

\section{Identification results based on MRI-radiomics signatures}

The radiomics signature was weighted with the regression coefficients for the signature construction presented in the form of a histogram in Fig. 2. Overall, there was a statistically significant difference observed in the average Rad-score between BOTs (Fig. 3) and malignancies in each of the selected MR-based radiomics models $(p<0.0001$, Table 3$)$. Table 4 illustrates the final classification results of the training data set and the validation data set. The model was first determined on the training data set based on the area under the ROC curve (AUC). Then, we evaluated the model on the validation data set. The coronal MR-based radiomics segmentation model yielded an accuracy of $78.9 \%$ to $82.8 \%$, while the sagittal model yielded an accuracy of $77.8 \%$ to $100 \%$. The 3D sagittal MR-based radiomics model yielded an ACC and an AUC of as high as $100 \%$ in differentiating between BOTs and malignancies in the validation data set (Table 4).

\section{Comparison of the performance results between the 2D and $3 \mathrm{D}$ radiomics models}

Considering two acquisition protocols, both coronal and sagittal MR-based features showed competitive accuracy in discriminating BOTs from malignancies either in 2D or 3D segmentation mode (2D AUC: 0.82 versus 0.84 and 3D AUC: 0.79 versus 1.0, respectively). 3D sagittal
fs-T2W images have the best performance compared to the other three methods in discriminating malignancies from BOTs, with an accuracy of $99 \%$ in the testing model. The ROC curve analysis with four kinds of segmentation methods in the validation group is summarized in Fig. 4.

\section{Discussion}

Ovarian BOT is a type of low-potential epithelial tumor with a relatively good prognosis after treatment. Sometimes, it is difficult to discriminate BOTs from ovarian malignancies solely on imaging information due to some overlapping imaging findings between the two [22]. Our current results showed that the 3D MR-based radiomics signatures derived from sagittal fs-T2WI yielded an ACC of $100 \%$ in differentiating ovarian malignancies from BOTs and may help clinicians make a correct diagnosis before surgery. To the best of our knowledge, this is the first reported study focusing on the diagnostic performance of MR-based radiomics signatures in ovarian tumor classification with $2 \mathrm{D}$ and $3 \mathrm{D}$ segmentation methods.

In the present study, the 3D signatures showed better performance than the $2 \mathrm{D}$ signatures did. This result can be easily appreciated because the 3D model utilized information of the whole lesion, more truly reflecting the tumoral heterogeneity than the 2D model did. The 

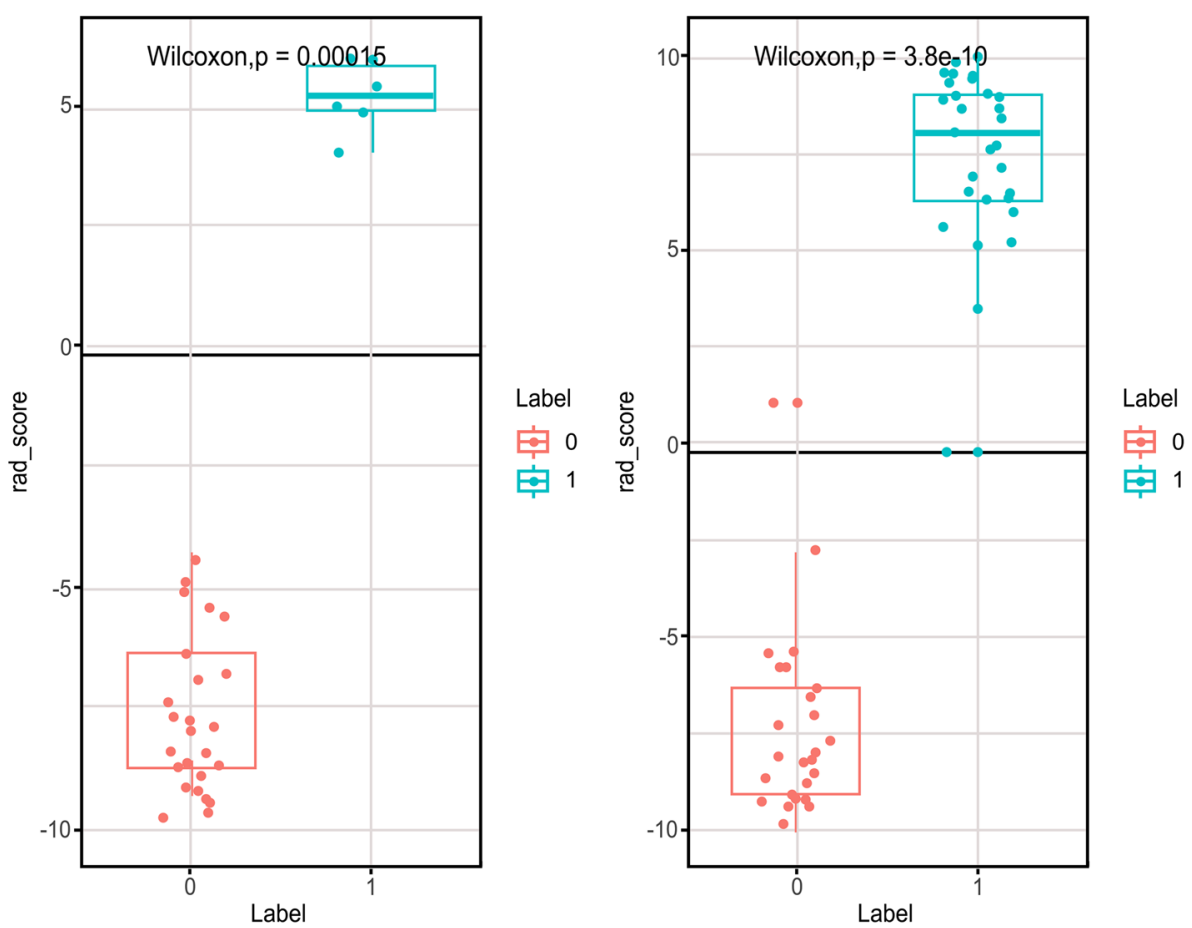

Fig. 3 The Stem-and-leaf plots of the average Rad-score in the LASSO model using 3D fs-sagittal T2WI radiomics signatures. Training group (left) and Testing group (right)

Table 3 The average Rad-score between BOT and malignancies in various MR-based radiomics models

\begin{tabular}{llll}
\hline Model & BOT $^{\mathbf{a}}$ & M & $\boldsymbol{P}^{\text {value }}$ \\
\hline 2D Coronal Training & $-0.73 \pm 0.88$ & $0.63 \pm 0.58$ & $<0.0001$ \\
2D Sagittal Training & $-0.63 \pm 0.78$ & $0.75 \pm 0.93$ & $<0.0001$ \\
3D Coronal Training & $-0.74 \pm 0.66$ & $0.87 \pm 1.34$ & $<0.0001$ \\
3D Sagittal Training & $-8.94 \pm 2.15$ & $9.55 \pm 2.4$ & $<0.0001$ \\
2D Coronal Testing & $-61.3 \pm 295.1$ & $0.22 \pm 0.90$ & $<0.0001$ \\
2D Sagittal Testing & $-0.19 \pm 3.28$ & $0.38 \pm 2.99$ & $<0.0001$ \\
3D Coronal Testing & $-0.66 \pm 0.76$ & $1.14 \pm 1.41$ & $<0.0001$ \\
3D Sagittal Testing & $-9.16 \pm 2.65$ & $8.89 \pm 2.47$ & $<0.0001$ \\
\hline
\end{tabular}

${ }^{a}$ mean $\pm s d$ current result is contrary to the previous CT radiomics study in which $2 \mathrm{D}$ radiomics features performed slightly better in non-small cell lung cancer prognostic estimation than 3D did [23]. The authors concluded that the reason might be related to the various axial $\mathrm{CT}$ image resolutions in their study in which the training and validation cohorts in the study sample were selected from different institutions.

Considering the two selected MRI protocols, the fs-sagittal sequences performed better than coronal sequences did on both 2D and 3D segmentation methods. Of note, the 3D-sagittal MR radiomics model yielded ACCs of $100 \%$ and $99 \%$ in the training and testing groups,

Table 4 The diagnostic performance in differentiating malignancies from BOT based on various MR-based radiomics models

\begin{tabular}{llllllll}
\hline Model & Group & SEN & SPE & PPV & NPV & ACC & AUC(95\% CI) \\
\hline 2d_cor & Training & 0.708 & 0.936 & 0.919 & 0.759 & 0.821 & $0.90(0.85-0.96)$ \\
2d_cor & Testing & 0.729 & 0.851 & 0.833 & 0.755 & 0.789 & $0.82(0.73-0.90)$ \\
3d_cor & Training & 0.875 & 0.717 & 0.764 & 0.846 & 0.798 & $0.85(0.77-0.93)$ \\
3d_cor & Testing & 0.936 & 0.717 & 0.772 & 0.917 & 0.828 & $0.84(0.76-0.93)$ \\
2d_sag & Training & 0.776 & 0.902 & 0.884 & 0.807 & 0.840 & $0.89(0.83-0.96)$ \\
2d_sag & Testing & 0.729 & 0.824 & 0.795 & 0.764 & 0.778 & $0.79(0.69-0.88)$ \\
3d_sag & Training & 1.000 & 1.000 & 1.000 & 1.000 & 1.000 & $1.0(1.0-1.0)$ \\
3d_sag & Testing & 1.000 & 0.980 & 0.980 & 1.000 & 0.990 & $1.0(1.0-1.0)$ \\
\hline
\end{tabular}

SEN sensitivity, SPE specificity, $P P V$ positive predictive value, NPV negative positive value, $A C C$ accuracy, $A U C$ area under the curve, $C l$ confidence interval 


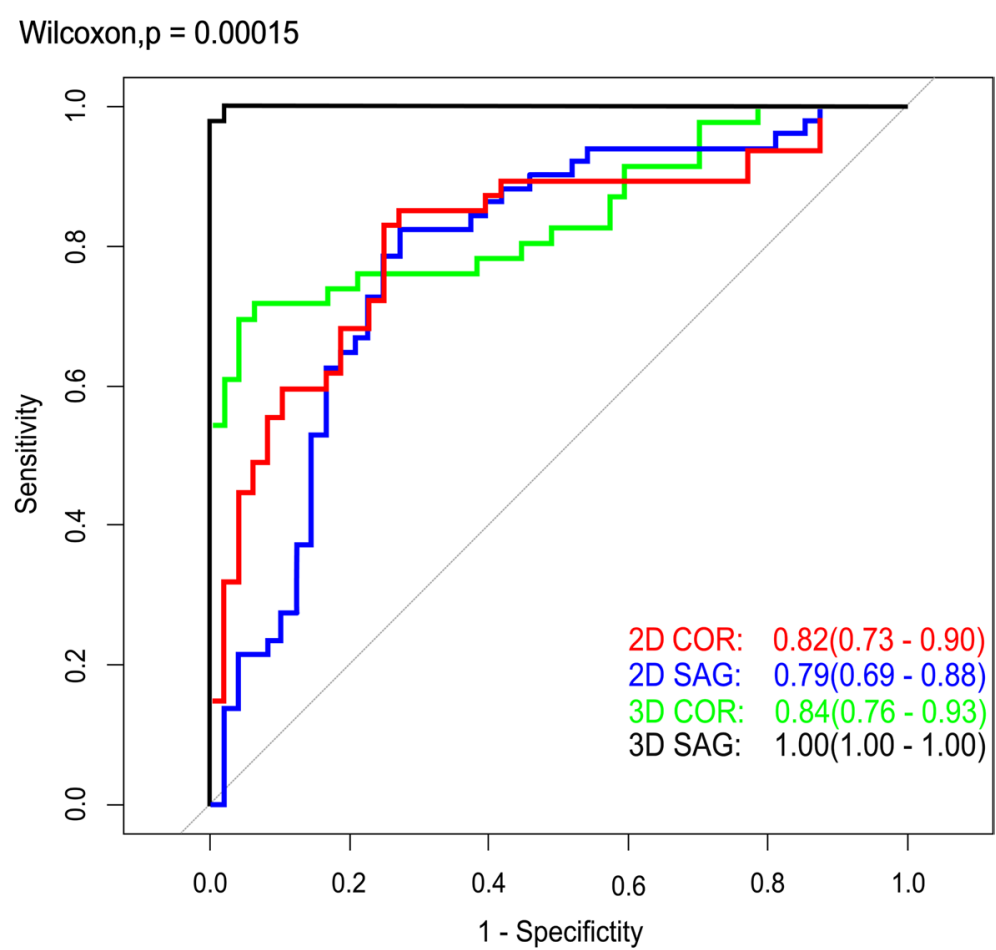

Fig. 4 ROC analysis of four kinds of MR-based radiomics signature models in determining ovarian malignancies from BOTs. Training group (A) and Testing group (B)

respectively. This finding is in accordance with our previous study in which fs-T2WI was also superior to coronal T2WI in Type I and Type II ovarian cancer categorization [5]. We believe that the sharp contrast between the lesion and the background on the fs MR sequence may play a role in the final determination. However, the true mechanism is unclear, and this result should also be validated in a future study with a large study sample.

Several radiomics studies using CT images have been reported for ovarian mass classification and prognostic estimation [24-27]. Fathi et al. found that the time-topeak and wash-in rate parameters showed a high SEN (89\% for the linear discriminant analysis [LDA] classifier and $97 \%$ for the support vector machine [SVM] classifier) and a high SPE (93\% for LDA and 100\% for SVM) in distinguishing malignancies from benign ovarian conditions among 55 sonographically indeterminate ovarian masses [26]. Qiu et al. acquired two sets of CT images (pretreatment and posttreatment) to compare three image features (tumor volume, tumor density, and density variance) between the two image sets in 30 ovarian cancer patients, and their model achieved an area under the curve of 0.831 in predicting progression-free survival when combining all three features together [25]. In this study, we used the LASSO method to establish the radiomics features model during the radiomics signature selection step as well as during the machine learning process. The Lasso model is reportedly a suitable method for analyzing a small sample with high-dimensional features due to its advantage of avoiding overfitting. A similar method was also reported in two recently published studies with promising results $[18,28]$.

There remains a limited number of studies on MRbased radiomics in ovarian tumor classification and posttreatment response prediction. In one study with 22 patients with advanced ovarian cancer, the authors found that apparent diffusion coefficient (ADC) values derived on the ADC map between primary ovarian cancer and metastatic sites differed significantly and may be used as response markers [29]. In the present study, we did not include DW images in the texture analysis. The lesion resolution on DWI, especially with large lesions, is relatively low, which is sometimes difficult to precisely outline in postprocess software. Moreover, in our previous study, we did not find that the ADC map could contribute more useful signatures in task classification than conventional MR images (T1W and T2W images) could [5]. Compared with traditional MRI analysis in differentiating BOTs from malignancies, radiomics signature results show better performance. In a traditional MRI reading session, the imaging signs always overlap with each other to some extent (for 
example, large size, solid components, irregular and thick septa) and lead to an inaccurate diagnosis [27, 30-32]. A recent study with proton MR spectroscopy (MRS) reported that the SEN and SPE were 91\% and $100 \%$ for solid components, respectively; additionally, the SEN and SPE were $84 \%$ and $82 \%$ for cystic components, respectively [12]. However, MRS scans are highly unit-dependent and time-consuming examinations and require operators with more experience than conventional methods do. From this point of view, radiomics signature analysis shows the potential clinical application owing to its simple segmentation step.

The limitations of this study included the fact that we did not include contrast-enhanced (CE) MR images to establish the MRI radiomics model. The CE-MRI scan was not available for all included patients in the current study, and therefore, we did not select this protocol for analysis to diminish the selection bias. Furthermore, in the present study, we only used conventional T2WI to establish a radiomics diagnostic model, which is different from the clinical reading scenario (mostly including T1WI, T2WI and DWI). Further study is necessary to explore the difference between one acquisition sequence and multiple acquisition sequences as in the clinical setting. In addition, all segmentation procedures were manually outlined on T2WI showing the best of the lesion; however, it is still an operatordependent procedure, and interoperator variation in segmentations may be emphasized, especially with multiple sequence images. Finally, all MR images were acquired in a 1.5-T MRI scanner, and a comparison study between 1.5-T and 3.0-T MRI machines should be validated in a large study in the future.

In summary, our results suggest that radiomics features that were extracted from T2W images were highly correlated with ovarian tumor subtype classification. 3D fs-sagittal MRI radiomics features may help clinicians differentiate ovarian BOTs from malignancies with high ACC.

\section{Abbreviations}

BOT: Borderline tumor; 2D: Two-dimensional; 3D: Three-dimensional; DWI: Diffusion weighted imaging; ADC: Apparent diffusion coefficient; PACS: Picture archiving and communication system; LASSO: Least absolute shrinkage and selection operator; LDA: Linear discriminant analysis; SVM: Support vector machine.

\section{Acknowledgements}

Not applicable

\section{Authors' contributions}

$H$. Zhang, K. Hua and H.Jiang designed of the whole study; H. Zhang, X. Liu and T. Wang performed the experiments and analyzed the data; $X$. Liu performed MRI scan and collected raw data; S.Duan analyzed the data and conducted statistical analysis; J. Jin provides the pathological data; G. Zhang and $\mathrm{H}$. Zhang revised and wrote the manuscript finally. All authors read and approved the final manuscript.

Funding

Not applicable

Availability of data and materials

The authors declare that all data supporting the findings of this study are available within the article.

\section{Declarations}

\section{Ethics approval and consent to participate}

Institutional Review Board approval was obtained.

This retrospective study was approved by institutional review board, and the requirement of the informed consent was waved.

- Retrospective.

- Observational in one institute.

\section{Consent for publication}

Written informed consent for publication was obtained from all participants.

\section{Competing interests}

The authors declare that they have no competing interests.

\section{Author details}

${ }^{1}$ Department of Radiology, Obstetrics and Gynecology Hospital, Fudan University, Shanghai, P.R. China. ${ }^{2}$ Department of Gynecology, Obstetrics and Gynecology Hospital, Fudan University, Shanghai, P.R. China. ${ }^{3} G E$ Healthcare, Shanghai, P.R. China. ${ }^{4}$ Department of Pathology, Obstetrics and Gynecology Hospital, Fudan University, Shanghai, P.R. China.

Received: 20 January 2021 Accepted: 31 December 2021

Published online: 03 February 2022

\section{References}

1. Fang C, Zhao L, Chen X, Yu A, Xia L, Zhang P. The impact of clinicopathologic and surgical factors on relapse and pregnancy in young patients ( $\leq 40$ years old) with borderline ovarian tumors. BMC Cancer. 2018;18(1):1147

2. Hauptmann S, Friedrich K, Redline R, Avril S. Ovarian borderline tumors in the 2014 WHO classification: evolving concepts and diagnostic criteria. Virchows Archiv : an international journal of pathology. 2017:470(2):125-42.

3. Prahm KP, Karlsen MA, Høgdall E, Scheller NM, Lundvall L, Nedergaard L, et al. The prognostic value of dividing epithelial ovarian cancer into type I and type II tumors based on pathologic characteristics. Gynecol Oncol. 2015:136(2):205-11.

4. Javadi S, Ganeshan DM, Qayyum A, Iyer RB, Bhosale P. Ovarian Cancer, the Revised FIGO Staging System, and the Role of Imaging. Am J Roentgenol. 2016:206(6):1351-60.

5. Zhang H, Mao Y, Chen X, Wu G, Liu X, Zhang P, et al. Magnetic resonance imaging radiomics in categorizing ovarian masses and predicting clinical outcome: a preliminary study. European Radiology. 2019;29(7):3358-71. https://doi.org/10.1007/s00330-019-06124-9.

6. Zhao SH, Qiang JW, Zhang GF, Ma FH, Cai SQ, Li HM, et al. Diffusionweighted MR imaging for differentiating borderline from malignant epithelial tumours of the ovary: pathological correlation. Eur Radiol. 2014:24(9):2292-9.

7. Li YA, Qiang JW, Ma FH, Li HM, Zhao SH. MRI features and score for differentiating borderline from malignant epithelial ovarian tumors. Eur J Radiol. 2018:98:136-42.

8. Thomassin-Naggara I, Daraï E, Cuenod CA, Rouzier R, Callard P, Bazot $M$. Dynamic contrast-enhanced magnetic resonance imaging: A useful tool for characterizing ovarian epithelial tumors. J Magn Reson Imaging 2008:28(1):111-20.

9. Thomassin-Naggara I, Balvay D, Aubert E, Daraï E, Rouzier R, Cuenod CA, et al. Quantitative dynamic contrast-enhanced MR imaging 
analysis of complex adnexal masses: a preliminary study. Eur Radiol. 2012;22(4):738-45.

10. Li H-H, Feng F, Qiang J-W, Zhang G-F, Zhao S-H, Ma F-H, et al. Quantitative dynamic contrast-enhanced MR imaging for differentiating benign, borderline, and malignant ovarian tumors. Abdominal Radiology. 2018:43(11):3132-41.

11. Li HMZS, Qiang JW, Zhang GF, Feng F, Ma FH, Li YA, Gu WY. Diffusion kurtosis imaging for differentiating borderline from malignant epithelial ovarian tumors: A correlation with Ki-67 expression. J Magn Reson Imaging. 2017:46(5):1499-506.

12. Ma FH, Li YA, Liu J, Li HM, Zhang GF, Qiang JW. Role of proton MR spectroscopy in the differentiation of borderline from malignant epithelial ovarian tumors: a preliminary study. J Magn Reson Imaging. 2019;49(6):1684-93.

13. Denewar FA, Takeuchi M, Urano M, Kamishima Y, Kawai T, Takahashi N, et al. Multiparametric MRI for differentiation of borderline ovarian tumors from stage I malignant epithelial ovarian tumors using multivariate logistic regression analysis. Eur J Radiol. 2017;91:116-23.

14 Shen D, Wu G, Zhang D, Suzuki K, Wang F, Yan P. Machine learning in medical imaging. Computerized Medical Imaging and Graphics. 2015;41 (Supplement C):1-2.

15. Gillies RJ, Kinahan PE, Hricak H. Radiomics: Images Are More than Pictures. They Are Data Radiology. 2016;278(2):563-77.

16. Gerges L, Popiolek D, Rosenkrantz AB. Explorative Investigation of WholeLesion Histogram MRI Metrics for Differentiating Uterine Leiomyomas and Leiomyosarcomas. Am J Roentgenol. 2018;210(5):1172-7.

17. Guo C-G, Ren S, Chen X, Wang Q-D, Xiao W-B, Zhang J-F, et al. Pancreatic neuroendocrine tumor: prediction of the tumor grade using magnetic resonance imaging findings and texture analysis with 3-T magnetic resonance. Cancer management and research. 2019;1 1:1933-44.

18. Zhang B, Tian J, Dong D, Gu D, Dong Y, Zhang L, et al. Radiomics Features of Multiparametric MRI as Novel Prognostic Factors in Advanced Nasopharyngeal Carcinoma. Clin Cancer Res. 2017;23(15):4259-69.

19. Nougaret $S$, Tardieu M, Vargas HA, Reinhold C, Vande Perre S, Bonanno $\mathrm{N}$, et al. Ovarian cancer: An update on imaging in the era of radiomics. Diagn Interv Imaging. 2019;100(10):647-55.

20. Altazi BA, Fernandez DC, Zhang GG, Hawkins S, Naqvi SM, Kim Y, et al. Investigating multi-radiomic models for enhancing prediction power of cervical cancer treatment outcomes. Physica Medica: European Journal of Medical Physics. 2018;46:180-8.

21. Zhang B, Ouyang F, Gu D, Dong Y, Zhang L, Mo X, et al. Advanced nasopharyngeal carcinoma: pre-treatment prediction of progression based on multi-parametric MRI radiomics. Oncotarget. 2017;8(42):72457-65.

22. Liu D, Zhang L, Indima N, Peng K, Li Q, Hua T, et al. CT and MRI findings of type I and type II epithelial ovarian cancer. Eur J Radiol. 2017;90:225-33.

23. Shen C, Liu Z, Guan M, Song J, Lian Y, Wang S, et al. 2D and 3D CT Radiomics Features Prognostic Performance Comparison in Non-Small Cell Lung Cancer. Translational oncology. 2017;10(6):886-94.

24 Rizzo S, Botta F, Raimondi S, Origgi D, Buscarino V, Colarieti A, et al. Radiomics of high-grade serous ovarian cancer: association between quantitative $C T$ features, residual tumour and disease progression within 12 months. European Radiology. 2018;28(11):4849-59.

25. Qiu Y, Tan M, McMeekin S, Thai T, Ding K, Moore K, et al. Early prediction of clinical benefit of treating ovarian cancer using quantitative CT image feature analysis. Acta radiologica(Stockholm, Sweden : 1987). 2016;57(9):1149-55.

26. Fathi KA, Mahrooz M, Hamidreza H, Sara P, Mahnaz N, Leila T, et al. Semiquantitative dynamic contrast-enhanced MRI for accurate classification of complex adnexal masses. J Magn Reson Imaging. 2017;45(2):418-27.

27. Vargas HA, Miccò M, Hong SI, Goldman DA, Dao F, Weigelt B, et al. Association between Morphologic CT Imaging Traits and Prognostically Relevant Gene Signatures in Women with High-Grade Serous Ovarian Cancer: A Hypothesis-generating Study. Radiology. 2015;274(3):742-51.

28. Vasquez MM, Hu C, Roe DJ, Chen Z, Halonen M, Guerra S. Least absolute shrinkage and selection operator type methods for the identification of serum biomarkers of overweight and obesity: simulation and application. BMC Med Res Methodol. 2016;16:154.

29. Sala E, Kataoka MY, Priest AN, Gill AB, McLean MA, Joubert l, et al. Advanced Ovarian Cancer: Multiparametric MR Imaging Demonstrates Response- and Metastasis-specific Effects. Radiology. 2012;263(1):149-59.
30. Feng W, Yuxiang W, Yan Z, Congrong L, Lizhi X, Zhenyu Z, et al. Comparison between types I and II epithelial ovarian cancer using histogram analysis of monoexponential, biexponential, and stretched-exponential diffusion models. J Magn Reson Imaging. 2017;46(6):1797-809.

31. Lalwani N, Prasad SR, Vikram R, Shanbhogue AK, Huettner PC, Fasih N. Histologic, Molecular, and Cytogenetic Features of Ovarian Cancers: Implications for Diagnosis and Treatment. Radiographics. 2011;31(3):625-46.

32. Sahdev A. CT in ovarian cancer staging: how to review and report with emphasis on abdominal and pelvic disease for surgical planning. Cancer Imaging. 2016;16:19.

\section{Publisher's Note}

Springer Nature remains neutral with regard to jurisdictional claims in published maps and institutional affiliations.
Ready to submit your research? Choose BMC and benefit from:

- fast, convenient online submission

- thorough peer review by experienced researchers in your field

- rapid publication on acceptance

- support for research data, including large and complex data types

- gold Open Access which fosters wider collaboration and increased citations

- maximum visibility for your research: over $100 \mathrm{M}$ website views per year

At BMC, research is always in progress.

Learn more biomedcentral.com/submissions 